\title{
AVALIAÇÃO DE SOLVENTES DE EXTRAÇÃO POR ULTRASSOM USANDO-SE CROMATOGRAFIA LÍQUIDA DE ALTA EFICIÊNCIA PARA A DETERMINAÇÃO DE HIDROCARBONETOS POLICÍCLICOS AROMÁTICOS EM SOLOS CONTAMINADOS
}

\author{
Jussara Aparecida Oliveira Cotta, Maria Olímpia Oliveira Rezende* e Maria Diva Landgraf \\ Instituto de Química de São Carlos, Universidade de São Paulo, Av. Trabalhador São Carlense, 400, 13560-970 São Carlos - SP, Brasil
}

Recebido em 22/7/08; aceito em 22/4/09; publicado na web em 1/10/09

\begin{abstract}
EVALUATION OF SOLVENT EXTRACTION BY ULTRASOUND BY USING HIGH PERFORMANCE LIQUID CHROMATOGRAPHY FOR THE DETERMINATION OF POLYCYCLIC AROMATIC HYDROCARBONS IN CONTAMINATED SOILS. A method using ultrasonication extraction for the determination of 17 polycyclic aromatic hydrocarbons (PAHs), selected by the USEPA and NIOSH as "consent decree" priority pollutants, in soil by High Performance Liquid Chromatography (HPLC) was studied. Separation and detection were completed in $20 \mathrm{~min}$ with a $\mathrm{C}_{18}$ columm, acetonitrile-water gradient elution and ultraviolet absorption and fluorescence detections. The detection limits, for a $10 \mu \mathrm{L}$ of solution injection, were less than $9,917 \mathrm{ng} / \mathrm{g}$ in UV detection and less than 1,866 ng/g in fluorescence detection. Several organic solvents were tested for extraction of the 17 PAHs from soils. Acetone was the best solvent among the three solvents tested, and the order of the extraction efficiencies was: acetone $>$ methanol>acetonitrile. Ultrasonication using acetone as solvent extraction was used to evaluate the biodegradation of those compounds in contaminated soil during a vermicomposting process.
\end{abstract}

Keywords: ultrasound extraction; solvent extraction; contaminated soils.

\section{INTRODUÇÃO}

Os hidrocarbonetos policíclicos aromáticos (HPAs) são compostos que possuem dois ou mais aneis benzênicos condensados em sua estrutura. ${ }^{1}$ Eles existem nos estados líquido ou sólido e apresentam ponto de ebulição maior do que $80^{\circ} \mathrm{C}$, sob pressão atmosférica normal. Sua ação carcinogênica tem principalmente sido observada em compostos tri-, tetra-, penta- e hexacíclicos. Os HPAs representam um importante grupo de micropoluentes orgânicos (xenobióticos) devido à alta capacidade de distribuição no ambiente (atmosfera, água e solo), ${ }^{1}$ sendo encontrados em matrizes ambientais como uma mistura extremamente complexa contendo numerosos isômeros em uma faixa extensa de concentração. ${ }^{2,3}$

Eles são produzidos pela combustão incompleta de combustíveis fósseis, bem como por processos diagenéticos durante a formação dos combustíveis fósseis; em pequenas quantidades por incêndios de florestas; possivelmente pela síntese microbiológica; pelos motores de exaustão a gasolina e, especialmente, os de combustão a diesel; o alcatrão da fumaça do cigarro; a superfície dos alimentos chamuscados ou queimados; fumaça da queima de madeira ou carvão e outros processos de combustão parcial, nos quais o carbono ou combustível não são convertidos em $\mathrm{CO}$ ou $\mathrm{CO}$. $^{4-6}$

Devido as suas propriedades físico-químicas, especialmente os HPAs de alta massa molar, são dificilmente degradáveis e tendem a se acumular em diferentes compartimentos ambientais. ${ }^{1,3,5,7-10}$ HPAs são considerados indicadores de contaminação por combustíveis, por não se degradarem no ambiente. Comparando-se os HPAs com menos de 4 aneis com os que contêm 4 ou mais aneis, estes últimos são menos voláteis e menos solúveis em água. Os HPAs permanecem no ambiente por um longo tempo. Por essa razão, é importante monitorar os HPAs tendo 4 ou mais aneis em amostras ambientais contaminadas por combustíveis. ${ }^{11}$ Os HPAs são adsorvidos nas partículas sólidas do solo, pois apresentam baixa solubilidade aquosa e alta hidrofobicidade. ${ }^{12}$ Embora existam centenas de HPAs no ambiente, somente 16 HPAs têm sido

*e-mail: mrezende@iqsc.usp.br selecionados pela United States Environmental Protection Agency (USEPA) e 17 HPAs pela National Institute for Occupational Safety and Health (NIOSH), para serem monitorados rotineiramente para fins reguladores. São eles: naftaleno, acenaftileno, acenafteno, fluoreno, fenantreno, antraceno, fluoranteno, pireno, benzo[a]antraceno, criseno, benzo[e]pireno, benzo[b]fluoranteno, benzo[k]fluoranteno, benzo[a] pireno, dibenzo[a,h]antraceno, benzo[g,h,i]perileno e indeno[1,2,3-cd] pireno. Devido a sua alta toxicidade carcinogênica e mutagênica e sua persistência no ambiente, ${ }^{13,14}$ a USEPA tem incluído os 16 HPAs em sua lista de poluentes prioritários e tem desenvolvido métodos para seu monitoramento no ambiente.

$\mathrm{Na}$ Europa apresenta-se uma lista de seis HPAs (fluoranteno, benzo[a]pireno, benzo[b]fluoranteno, benzo[k]fluoranteno, benzo[g,h,i]perileno e indeno(1,2,3-cd pireno) para monitoramento em águas superficiais usada para fins de potabilidade. Em 1986, a USEPA propôs uma regulamentação para diminuir riscos de poluição, incluindo limites de concentração para poluentes orgânicos como benzo[a]pireno, por exemplo.

Os melhores métodos disponíveis para monitorar HPAs são a cromatografia líquida (CL) com detecção por fluorescência e ultravioleta e a cromatografia gasosa acoplada ao espectrômetro de massas. As etapas de separação e detecção para amostras sólidas são descritas no método 8310 da EPA, usando CL com detecção no ultravioleta (UV) e fluorescência e método 8100, usando CG acoplada a espectrômetro de massas. Os vários métodos já validados não deixam dúvidas quanto às vantagens da CL no fracionamento de misturas complexas de HPAs e no clean-up de amostras. Por exemplo, os procedimentos analíticos recomendados pela USEPA são documentados nos métodos 550.1 (água potável), 610 (água residual), 8310 (resíduo sólido) e TO-13 (ar). Todos esses métodos são baseados na CL com detector UV e fluorescência. A CLAE - cromatografia líquida de alta eficiência-é preferível à cromatografia gasosa de alta resolução (CGAR), pois mesmo que apresente uma eficiência mais baixa na resolução de HPAs com baixa massa molar, as colunas de fase reversa podem realmente separar um número de isômeros de HPAs que são difíceis de separar por CGAR. ${ }^{15}$ Para cada método, uma etapa de extração e concentração é requerida e 
o principal problema para análise de traços de poluentes orgânicos vem da complexidade das várias matrizes, que depende de sua origem. ${ }^{12}$

A extração do analito em amostras sólidas continua sendo um passo crítico na análise de contaminantes. Deve-se avaliar o método de extração sempre levando em conta os seguintes aspectos: seletividade para os componentes de interesse, recuperação do analito, volume do solvente orgânico necessário, toxicidade do solvente, tempo de extração e número de passos de clean-up requeridos após a extração. ${ }^{16,17}$ Cada técnica tem seu próprio mérito e a escolha da extração depende, ainda, de outros fatores como o custo de capital, o custo e a simplicidade de operação e a disponibilidade de um método padrão ou validado. Existe uma série de técnicas de extração por solventes comumente usadas para a extração de hidrocarbonetos em solo e sedimento. ${ }^{17-20}$ Há procedimentos de extração que incluem Soxhlet, ultrassom, agitação mecânica, refluxo com $\mathrm{KOH} \mathrm{e}$ destilação a vapor, e técnicas que incluem extração por fluido supercrítico, extração líquida pressurizada, entre outras. ${ }^{21}$ A extração em Soxhlet é o método recomendado pela USEPA para a extração de compostos orgânicos semi-volatéis e não voláteis de matrizes sólidas. Esta extração, cuja eficiência é alta, tem sido por muitos anos o método padrão para preparar um extrato de solvente das matrizes sólidas contendo HPAs. Contudo, o procedimento é tedioso, pois o tempo de extração é longo, com aproximadamente $16 \mathrm{~h}$ ou mais, requer uma grande quantidade de solvente e pode, ainda, degradar compostos termicamente lábeis. ${ }^{22}$ Mais recentemente, outras técnicas de extração em amostras ambientais sólidas têm sido desenvolvidas para tentar reduzir o tempo de extração e a quantidade de solvente como, por exemplo, por ultrassom. Em comparação à extração por Soxhlet, a extração por ultrassom ocorre em curto espaço de tempo (cerca de $15 \mathrm{~min}$ ) e oferece boa recuperação dos analitos, por meio de um equipamento simples e de fácil operação. ${ }^{23}$ A otimização dos parâmetros de extração por ultrassom, incluindo tipo de solvente ou composição do solvente, tempo de extração, carga da amostra e teor de água, é necessária para a obtenção de uma maior eficiência e reprodutibilidade de extração. ${ }^{5}$ Além disso, para melhorar a recuperação obtida, usa-se um solvente polar, como acetona ou metanol, minimizando-se, assim, a necessidade da secagem das amostras antes da extração. A secagem de amostras contendo analitos voláteis ou semi-voláteis é um fator de erro, devido à perda desses analitos. Até cerca de $16 \%$ de perdas de hidrocarbonetos têm sido observadas, em consequência da secagem das amostras em forno a $45^{\circ} \mathrm{C}$. O uso de altas temperaturas na extração em Soxhlet também resulta em perdas de hidrocarbonetos atribuídas à volatilização e/ou oxidação de espécies altamente voláteis e termodinamicamente lábeis. Vários estudos têm sido relatados ${ }^{22,23}$ mostrando a eficiência de extração por ultrassom de analitos orgânicos em sedimento e solo utilizando diferentes solventes.

Os objetivos deste trabalho foram adaptar, otimizar e validar a técnica de extração por ultrassom para análise de HPAs em solo, em nível de $\mathrm{mg} \mathrm{kg}^{-1}$ (matéria seca), utilizando-se CLAE/ fluorescência e CLAE/UV/Vis com limites de detecção de HPAs em níveis de $\mu \mathrm{g}$ $\mathrm{L}^{-1}$ a ng $\mathrm{L}^{-1}$ para cada composto e aperfeiçoar o método de extração utilizando-se solventes com diferentes polaridades. As melhores condições de extração e análise simultânea dos 17 HPAs foram otimizadas e posteriormente validadas com relação às principais figuras de mérito: exatidão precisão, limite de detecção e quantificação, linearidade, sensibilidade e seletividade. A técnica otimizada e validada foi utilizada para avaliar a biodegradação destes compostos durante um processo de vermicompostagem.

\section{PARTE EXPERIMENTAL}

\section{Reagentes e soluções padrão}

Todos os reagentes e solventes utilizados foram de grau analítico. $\mathrm{O}$ sal de sulfato de sódio foi obtido da Mallinckrodt Chemicals. Os solventes acetona, acetonitrila e metanol foram, também, obtidos da Mallinckrodt Chemicals; os padrões sólidos dos correspondentes HPAs da Supelco.

As soluções padrão dos HPAs foram preparadas separadamente em uma concentração de $100 \mathrm{mg} \mathrm{L}^{-1} \mathrm{em}$ acetonitrila. A soluçãoestoque padrão ou padrão mista foi preparada pela dissolução de quantidades apropriadas dos HPAs em acetonitrila, dependendo da resposta de cada HPA pelo detector de fluorescência e o acenaftileno pelo UV, com as seguintes concentrações: naftaleno $\left(0,492 \mathrm{mg} \mathrm{L}^{-1}\right)$, acenaftileno $\left(0,984 \mathrm{mg} \mathrm{L}^{-1}\right)$, acenafteno $\left(0,032 \mathrm{mg} \mathrm{L}^{-1}\right)$, fluoreno $\left(0,162 \mathrm{mg} \mathrm{L}^{-1}\right)$, fenantreno $\left(0,540 \mathrm{mg} \mathrm{L}^{-1}\right)$, antraceno $\left(0,032 \mathrm{mg} \mathrm{L}^{-1}\right)$, fluoranteno $\left(3,142 \mathrm{mg} \mathrm{L}^{-1}\right)$, pireno $\left(0,212 \mathrm{mg} \mathrm{L}^{-1}\right)$, benzo[a] antraceno $\left(0,236 \mathrm{mg} \mathrm{L}^{-1}\right)$, criseno $\left(0,540 \mathrm{mg} \mathrm{L}^{-1}\right)$, benzo[e]pireno $(0,687 \mathrm{mg}$ $\left.\mathrm{L}^{-1}\right)$, benzo[e]acefenantrileno ou benzo[b]fluoranteno $\left(0,491 \mathrm{mg} \mathrm{L}^{-1}\right)$, benzo[k]fluoranteno $\left(0,039 \mathrm{mg} \mathrm{L}^{-1}\right)$, benzo[a]pireno $\left(0,130 \mathrm{mg} \mathrm{L}^{-1}\right)$, dibenzo[a,h]antraceno $\left(1,944 \mathrm{mg} \mathrm{L}^{-1}\right)$, benzo[g,h,i]perileno $(1,296$ $\left.\mathrm{mg} \mathrm{L}^{-1}\right)$ e indeno[1,2,3-cd]pireno $\left(1,296 \mathrm{mg} \mathrm{L}^{-1}\right)$. As soluções padrão foram estocadas sob refrigeração a $4{ }^{\circ} \mathrm{C}$.

As soluções padrão de trabalho foram preparadas por diluição da solução-estoque a um volume final de $5,0 \mathrm{~mL}$ com acetonitrila. A identificação dos HPAs foi feita em relação aos tempos de retenção dos picos e à quantificação pelas áreas dos mesmos.

\section{Amostragem}

O solo utilizado foi coletado na fazenda Santa Isabel, na Rodovia SP215, km 140, município de São Carlos, estado de São Paulo, caracterizando um solo não cultivado e sem histórico de contaminação. Na Tabela 1 são apresentadas as características do solo.

Tabela 1. Caracterização física e química do solo utilizado

\begin{tabular}{|c|c|}
\hline Características & Valores \\
\hline $\mathrm{pH}^{\mathrm{a}}$ & $4,51 \pm 0,01$ \\
\hline Carbono orgânico $\left(\mathrm{g} \mathrm{kg}^{-1}\right)^{\mathrm{b}}$ & $8,55 \pm 0,30$ \\
\hline Umidade $(\%)^{\mathrm{c}}$ & $7,012 \pm 0,090$ \\
\hline Teor de matéria orgânica $(\%)^{\mathrm{d}}$ & $1,463 \pm 0,052$ \\
\hline CTC $\left(\mathrm{cmol}_{\mathrm{c}} \mathrm{kg}^{-1}\right)^{\mathrm{e}}$ & $1,32 \pm 0,00$ \\
\hline $\operatorname{Argila}(\%)^{\mathrm{f}}$ & 3,3 \\
\hline Silte $(\%)^{\mathrm{f}}$ & 6,7 \\
\hline $\operatorname{Areia}(\%)^{\mathrm{f}}$ & 90 \\
\hline
\end{tabular}

aDeterminação da atividade hidrogeniônica através de uma suspensão com 25,00 mL de solução de cloreto de cálcio $0,01 \mathrm{~mol} \mathrm{~L}^{-1}$ e $10,00 \mathrm{~g}$ de

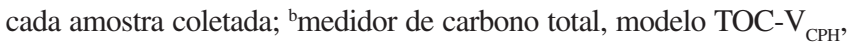
acoplado ao módulo de amostras sólidas, modelo SSM-5000A, marca Shimadzu, com detector de combustão; ' em estufa a 60-65 ${ }^{\circ} \mathrm{C}$ e a $100-110$

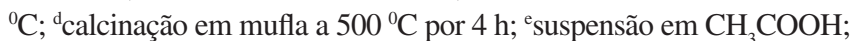
fjogo de peneiras de nos. 16, 40, 60, 100 e 230, as quais foram agitadas em mesa agitadora por $10 \mathrm{~min}$. As determinações da fração silte e argila foram feitas pelo método do densímetro, da norma técnica (NBR).

\section{Instrumentação}

A determinação dos HPAs foi feita por CLAE, utilizando-se um cromatógrafo da marca Shimadzu, com detector UV-Vis, modelo SPD-10A VP, e fluorescência, modelo RF-10A, utilizando uma coluna C-18 (250 x 4,6 mm SS, Wakosil II 5C18AR $5 \mu \mathrm{m}$, SGE). A acetonitrila e a água deionizada foram usadas como eluentes na vazão de $1,8 \mathrm{~mL} \mathrm{~min}^{-1}$. O gradiente de eluição foi de 0-9,6 min: acetonitrila-(25-75\%) água, 9,6-20 min: $100 \%$ acetonitrila. A temperatura da coluna foi mantida a $25^{\circ} \mathrm{C}$. O comprimento de onda de 
excitação/emissão na fluorescência foi fixado em: $280 / 320 \mathrm{~nm}$ a 0,01 $\min ; 240 / 398 \mathrm{~nm}$ a $9,6 \mathrm{~min}, 300 / 466 \mathrm{~nm}$ a $18,10 \mathrm{~min}, 240 / 398 \mathrm{~nm}$ a 19,8 min até $20 \mathrm{~min}$. Quando a determinação foi feita por UV-Vis, o comprimento de onda foi fixado em $254 \mathrm{~nm}$.

Todos os parâmetros para a extração e as condições cromatográficas foram otimizados para obtenção da melhor e mais eficiente separação dos compostos estudados.

\section{Extração da amostra e recuperação}

Para a avaliação do método foram utilizadas amostras do solo como matriz, adicionando-se soluções padrão de concentração conhecida dos HPAs (maior nível de concentração encontrado na Tabela 2). Essas matrizes foram, então, submetidas ao mesmo procedimento analítico para as amostras reais, sendo avaliadas a porcentagem de recuperação do método e desvio padrão relativo.

Para o método de extração de HPAs foi utilizada a técnica de ultrassom. As extrações foram realizadas em triplicata e de cada extrato foram feitas 5 injeções cromatográficas. A recuperação do método de extração foi determinada comparando-se o cromatograma da mistura de HPAs no solo dopado com solução padrão com o cromatograma do padrão na respectiva concentração, ambos submetidos aos mesmos procedimentos de extração.

Cinco gramas das amostras e $5 \mathrm{~g}$ de sulfato de sódio anidro foram colocados em recipiente fechado de $200 \mathrm{~mL}$ com $25 \mathrm{~mL}$ de solvente. A amostra foi sonicada em banho de ultrassom (modelo 2510, marca Bransom) por 15 min. A solução extraída foi centrifugada e filtrada em um vial (em filtro Minisart RC15 de $15 \mathrm{~mm}$ de diâmetro e 0,45 $\mu \mathrm{m}$ de porosidade da marca Sartorius) e armazenada em refrigerador a $4{ }^{\circ} \mathrm{C}$ para posterior determinação.

\section{Polaridade do solvente extrator}

Foi avaliada a porcentagem de recuperação dos HPAs utilizandose solventes com diferentes polaridades. Para tanto, foram escolhidos como solvente extrator acetona, metanol e acetonitrila.

\section{Validação do método}

A metodologia otimizada foi validada com relação aos seguintes parâmetros: linearidade, limite de detecção e quantificação, precisão (repetibilidade) e exatidão (ensaio de recuperação).

A linearidade da resposta foi avaliada submetendo-se amostras de solo fortificadas com padrão dos HPAs. Os limites de detecção e quantificação foram calculados pelos desvios-padrão dos valores de y no ponto de intercepto com a regressão linear divididos pelos coeficientes angulares das curvas analíticas; este valor é multiplicado por 3 para se determinar o limite de detecção (LOD) e por 10 para o de quantificação (LOQ) ${ }^{24}$

$$
\mathrm{LOD}=3 \cdot(\sigma / \mathrm{A}) \quad \mathrm{LOQ}=10 \cdot(\sigma / \mathrm{A})
$$

onde: $\sigma=$ desvio-padrão dos valores de y no ponto de intercepto com a regressão linear; $\mathrm{A}=$ coeficiente angular da curva analítica.

A precisão do método foi determinada nas amostras de solo com 5 repetições. Já a exatidão do método foi avaliada, em triplicata, por ensaios de recuperação.

\section{RESULTADOS E DISCUSSÃO}

\section{Separação analítica dos HPAs}

O método 8310 da USEPA ${ }^{25}$ propõe determinar os compostos naftaleno, acenaftileno, acenafteno e fluoreno pelo detector UV-Vis e os demais por fluorescência. Mas pôde-se observar, após uma varredura entre 200 e $600 \mathrm{~nm}$, em um espectrofotômetro UV-VisNIR (Cary 5G, Varian), seguida de uma varredura entre 250 e 550 $\mathrm{nm}$ em um comprimento de onda de excitação estabelecido em um espectrofotômetro de fluorescência (F-4500, Hitachi) para todos os compostos, que o naftaleno, o acenafteno e o fluoreno têm uma boa detecção por fluorescência.

Observando-se as Figuras 1 e 2, nota-se que o melhor comprimento de onda de excitação para os HPAs naftaleno, acenafteno e fluoreno seria próximo a $224 \mathrm{~nm}$. O estudo desses compostos seria prejudicado em uma faixa de comprimento de onda de excitação/ emissão em que respondem ( $\lambda_{\mathrm{ex}}=224 \mathrm{~nm}$ e $\lambda_{\mathrm{em}}=330 \mathrm{~nm}$ ), pois há um aumento significativo na linha de base do cromatograma, o que impede a correta integração dos picos. Devido a isso, utilizou-se $\lambda_{\mathrm{ex}}$ $=280 \mathrm{~nm}$ e $\lambda_{\text {em }}=330 \mathrm{~nm}$ para o naftaleno, acenafteno e fluoreno.

Apesar de se registrar um sinal do acenaftileno da mesma ordem de grandeza dos demais compostos, deve-se ressaltar que sua concentração foi cerca de 20 vezes maior em comparação à concentração dos demais, pois este composto fluoresce muito pouco. ${ }^{23}$

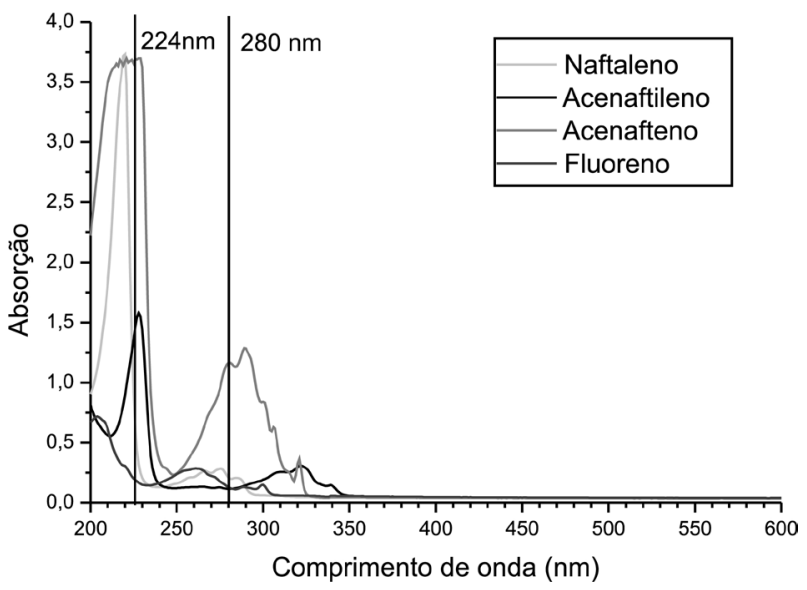

Figura 1. Espectro UV-Vis do naftaleno, acenaftileno, acenafteno e fluoreno

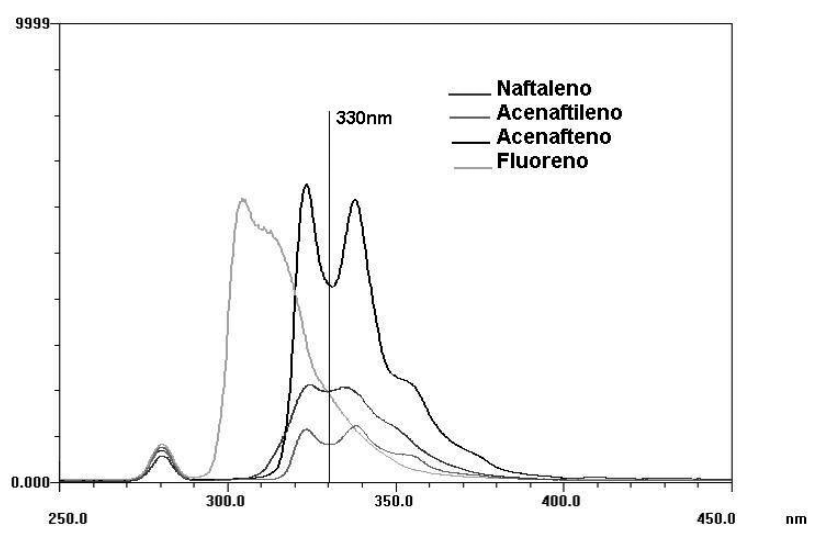

Figura 2. Espectro de fluorescência do naftaleno, acenaftileno, acenafteno e fluoreno $\left(l_{e x}=280 \mathrm{~nm}\right)$

Sabe-se que cada HPA apresenta uma melhor detecção em um comprimento de onda específico, mas não foi possível utilizar o comprimento de onda ótimo para cada composto devido ao grande número de compostos e à proximidade de alguns deles na ordem de eluição, ou seja, tempo de retenção muito próximo. Mas neste caso, estipulou-se um comprimento de onda de emissão e excitação próximos ao ideal para todos. 
Foi então verificado o melhor comprimento de onda que se deveria utilizar para cada HPA no detector de fluorescência, a fim de se obter a melhor sensibilidade para todos os analitos. As melhores condições observadas, nas quais foram obtidas as curvas analíticas e toda metodologia analítica, foram baseadas nos dados de Varian Chromatography Systems e Andrade. ${ }^{26,27}$

Para o indeno(1,2,3,cd)pireno alcançou-se uma boa detecção no comprimento de onda de excitação em 300 nm e emissão em 466 nm e os demais HPAs no comprimento de onda de excitação em $240 \mathrm{~nm}$ e emissão em $398 \mathrm{~nm}$.

Por esta razão, o comprimento de onda de excitação/emissão na fluorescência foi fixado em $280 / 330 \mathrm{~nm}$ a $0,01 \mathrm{~min} ; 240 / 398 \mathrm{~nm}$ a 9,6 $\min , 300 / 466 \mathrm{~nm}$ a $18,10 \mathrm{~min}$ e $240 / 398 \mathrm{~nm}$ a $19,8 \mathrm{~min}$ até $20 \mathrm{~min}$.

Utilizaram-se os detectores de UV-Vis e de fluorescência em linha (online), criando-se o método com intenção de quantificar o composto acenaftileno utilizando o detector UV-Vis e para os compostos naftaleno, acenafteno, fluoreno, fenantreno, antraceno, fluoranteno, pireno, benzo[a]antraceno, criseno, benzo[e]pireno, benzo[k]fluoranteno ou benzo[e]acefenantrileno, benzo[k]fluoranteno, benzo[a]pireno, dibenzo[a,h]antraceno, benzo[g,h,i]perileno, indeno[1,2,3-cd]pireno utilizando-se o detector de fluorescência, pois estes apresentam maior sensibilidade nesse detector.

Pelos valores obtidos, verificou-se que o limite de detecção do naftaleno e acenafteno + fluoreno é 56 e 5 vezes menor no detector de fluorescência em relação ao UV-Vis, respectivamente. Nota-se, assim, que há uma maior sensibilidade destes compostos no detector de fluorescência. Por isso, resolveu-se determinar o naftaleno e acenafteno + fluoreno via detector de fluorescência, modificando-se, assim, ligeiramente o método 8310 proposto pela USEPA.

\section{Separação cromatográfica}

Cromatogramas com detecção por UV-Vis (Figura 3) e por fluorescência (Figura 4) nas melhores condições cromatográficas foram obtidos pela injeção direta da mistura-padrão dos 17 HPAs. A separação dos 17 HPAs foi alcançada em $20 \mathrm{~min}$. Somente o acenaftileno, por não fluorescer, não foi detectado no detector de fluorescência. Em nenhuma das condições de eluição foi possível separar os pares acenafteno/fluoreno.

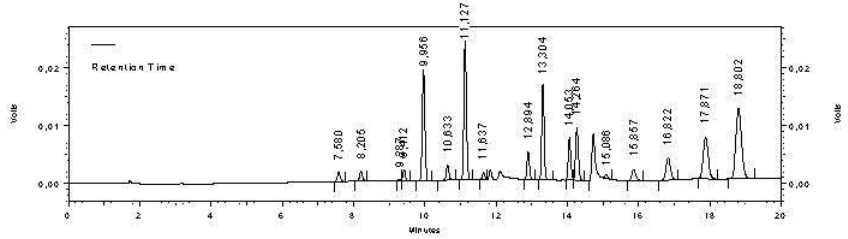

Figura 3. Cromatograma obtido pelo detector de UV-Vis (para identificação dos HPAs ver Tabela 2)

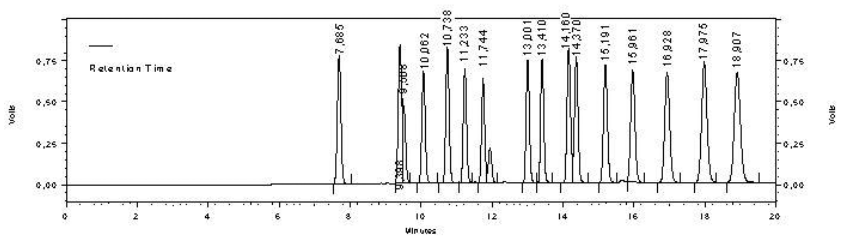

Figura 4. Cromatograma obtido pelo detector fluorescência (para identificação dos HPAs ver Tabela 2)

\section{Linearidade, sensibilidade e precisão}

A cromatografia líquida com detectores de fluorescência e UV fornece uma resposta linear para quantidades injetadas na faixa pré-estabelecida para cada composto estudado. Como exemplo, apresenta-se na Figura 5 a curva para naftaleno. A relação da concentração com a área do pico foi usada para avaliar a linearidade, sensibilidade e precisão do método. As curvas analíticas obtidas, através de suas respectivas equações das retas, permitiram o cálculo dos coeficientes de determinação, desvio-padrão, limite de detecção e limite de quantificação do equipamento.

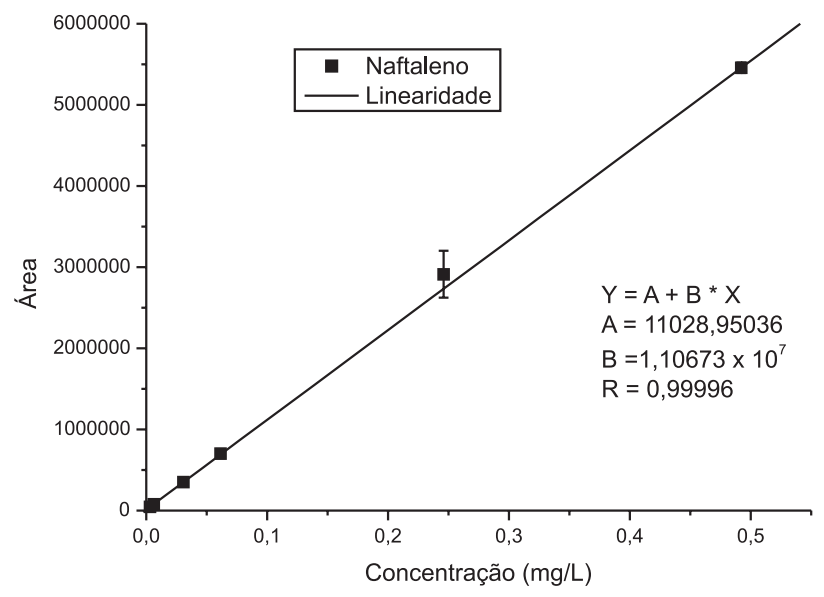

Figura 5. Curva analítica do naftaleno

A Tabela 2 apresenta o intervalo no qual se estudou a linearidade do sistema para os 17 HPAs, além dos respectivos resultados que mostram a sensibilidade e precisão do método. Os HPAs apresentaram boa linearidade, com valores de coeficiente de determinação na faixa de 0,99965-0,99999. Os limites de detecção (Tabela 2) obtidos por injeção direta da mistura-padrão dos 17 HPAs foram calculados com base na definição da IUPAC, pelas curvas analíticas de cada composto, e ficaram entre 3 e 74 vezes menores usando-se detecção por fluorescência com relação aos valores comparados com detecção por UV. Com as condições experimentais aqui relatadas, o limite de detecção no detector de fluorescência está na faixa de $7,1 \times 10^{-6} \mathrm{mg}$ $\mathrm{L}^{-1}$, injetado para o antraceno, a $1,9 \times 10^{-3} \mathrm{mg} \mathrm{L}^{-1}$ para o fluoranteno e os limites de detecção com UV (254 nm) na faixa de $4,5 \times 10^{-4} \mathrm{mg} \mathrm{L}^{-1}$ para o benzo[e]pireno a $9,9 \times 10^{-3} \mathrm{mg} \mathrm{L}^{-1}$ para o acenaftileno.

\section{Seleção de solvente para extração}

Como individualmente os HPAs podem estar presentes no solo em níveis de ng $\mathrm{g}^{-1}$ ou menos, poucas matrizes podem ser determinadas diretamente sem interferentes sérios. Então, uma extração eficiente, pré-concentração e clean-up das amostras são operações imprescindíveis para a determinação de HPAs.

Sun et al. utilizaram acetona, cicloexano, hexano, diclorometano, tolueno ou mistura destes solventes para a extração de HPAs e observaram grandes diferenças no percentual de recuperação. ${ }^{23}$ Song et al. relataram valores de recuperações adequados na extração de HPAs em solo com $98 \%$ de areia usando ultrassom. ${ }^{18} \mathrm{Na}$ maioria dos trabalhos publicados, os HPAs mais voláteis, com 2 ou 3 aneis aromáticos, tais como naftaleno e acenaftileno, alcançaram valores de recuperação não reprodutíveis, porque o solvente usado para extração e eluição, na extração por Soxhlet, por exemplo, foi evaporado quase à secura usando-se um evaporador rotatório e fluxo de nitrogênio. A fim de evitar perdas dos HPAs mais voláteis e aumentar a eficiência de extração, uma seleção apropriada dos solventes foi empregada para otimizar o procedimento de extração dos 17 HPAs, sem que houvesse a etapa de evaporação do solvente. Neste trabalho, três diferentes solventes (acetona, metanol e acetonitrila) foram testados. Os resultados para as recuperações, usando 


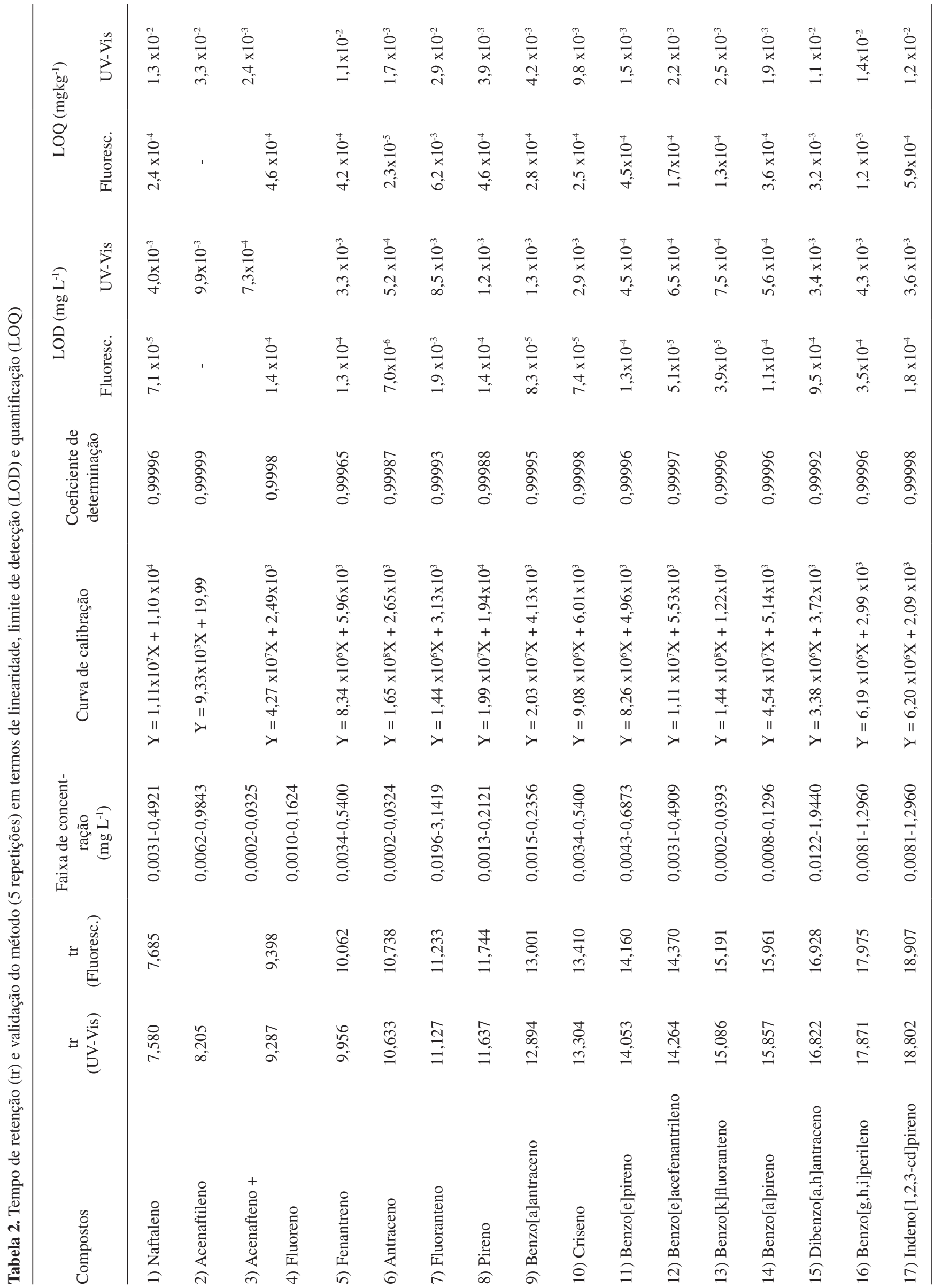


Tabela 3. Recuperação (R) e desvio padrão relativo (DPR) para extração por ultrassom de solo com metanol, acetonitrila e acetona como solventes (em triplicata)

\begin{tabular}{|c|c|c|c|c|c|c|}
\hline \multirow{2}{*}{ HPAs } & \multicolumn{2}{|c|}{ metanol } & \multicolumn{2}{|c|}{ acetonitrila } & \multicolumn{2}{|c|}{ acetona } \\
\hline & $\mathrm{R}(\%)$ & $\operatorname{DPR}(\%)$ & $\mathrm{R}(\%)$ & $\operatorname{DPR}(\%)$ & $\mathrm{R}(\%)$ & $\operatorname{DPR}(\%)$ \\
\hline 1) Naftaleno & 62,2 & 4,5 & 50,1 & 4,4 & 82,2 & 2,5 \\
\hline 2) Acenaftileno & 65,2 & 2,9 & 97,8 & 11,1 & 59,1 & 3,6 \\
\hline 3) Acenafteno + 4) Fluoreno & 57,7 & 9,8 & 58,8 & 9,3 & 75,1 & 1,0 \\
\hline 5) Fenantreno & 70,0 & 3,5 & 64,0 & 6,4 & 91,2 & 4,6 \\
\hline 6) Antraceno & 58,5 & 2,3 & 67,1 & 7,9 & 74,0 & 2,7 \\
\hline 7) Fluoranteno & 72,4 & 1,5 & 71,4 & 6,5 & 80,0 & 2,2 \\
\hline 8) Pireno & 73,4 & 4,5 & 65,9 & 5,8 & 88,9 & 3,3 \\
\hline 9) Benzo[a]antraceno & 74,8 & 2,3 & 73,1 & 9,6 & 83,4 & 4,2 \\
\hline 10) Criseno & 78,2 & 2,3 & 73,4 & 9,8 & 88,7 & 4,6 \\
\hline 11) Benzo[e]pireno & 74,8 & 2,0 & 69,6 & 9,2 & 82,8 & 2,5 \\
\hline 12) Benzo[e]acefenantrileno & 63,5 & 1,3 & 73,3 & 9,9 & 78,9 & 1,9 \\
\hline 13) Benzo[k]fluoranteno & 76,6 & 1,4 & 72,3 & 8,4 & 78,9 & 0,8 \\
\hline 14) Benzo[a]pireno & 20,5 & 9,1 & 46,3 & 13,9 & 46,0 & 6,2 \\
\hline 15) Dibenzo[a,h]antraceno & 70,0 & 3,0 & 70,3 & 6,9 & 76,2 & 1,4 \\
\hline 16) Benzo[g,h,i]perileno & 67,1 & 4,7 & 69,1 & 6,0 & 73,4 & 4,6 \\
\hline 17) Indeno[1,2,3-cd]pireno & 66,6 & 5,4 & 49,4 & 10,3 & 73,0 & 7,8 \\
\hline
\end{tabular}

o procedimento de extração em ultrassom, são mostrados na Tabela 3. A precisão do método, representada como desvio-padrão relativo (DPR) para determinação de replicatas, foi menor que $10,0 \%$, exceto para acenaftileno, que foi de $11,1 \%$ e benzo[a]pireno de $13,9 \%$, ambos utilizando acetonitrila como solvente de extração. Foram observadas grandes diferenças no percentual de recuperação quando esses diferentes solventes foram utilizados para a extração de HPAs no solo.

Como mostrado na Figura 6, a eficiência da extração para os 17 HPAs no solo com acetona foi definida como 1, sendo as demais representadas por uma razão dos sinais obtidos pelos outros solventes, em comparação com os sinais obtidos pela acetona. Na Figura 6 observa-se que as mais elevadas eficiências de extração para a maioria dos 17 HPAs, em relação aos três solventes utilizados, foram obtidas usando acetona, exceto para o acenaftileno.

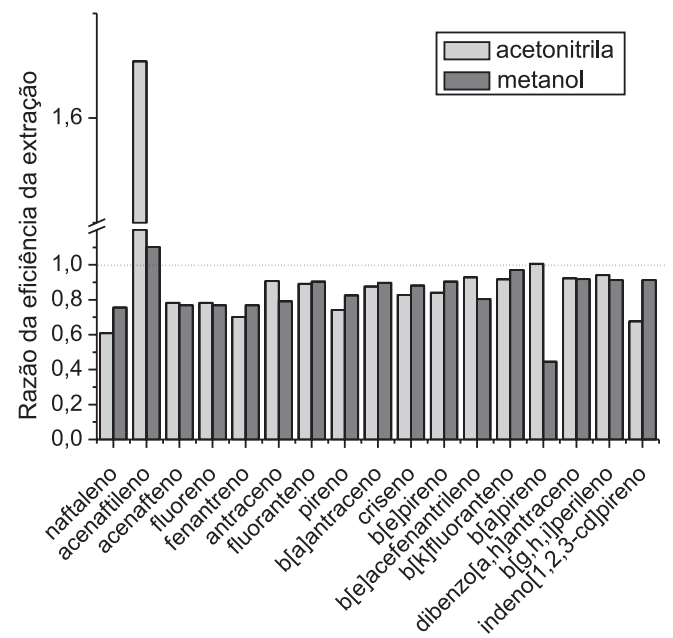

Figura 6. Seleção do solvente para extração dos 17 HPAs. O eixo vertical é a razão dos sinais para os HPAs extraídos do solo pelos outros solventes comparativamente ao obtido com a acetona
A ordem de eficiência de extração para a maioria dos 17 HPAs do solo pelos solventes foi acetona $>$ metanol $>$ acetonitrila. Como a mistura acetonitrila-água foi o solvente usado como fase móvel e levando-se em conta que o solvente que é miscível com a fase móvel é o melhor para a solução de injeção final, selecionou-se a acetona como o solvente de extração. A etapa de evaporação do solvente quase à secura pôde ser eliminada, pois a sensibilidade foi bastante alta para a determinação dos HPAs pelo método proposto. Além disso, com acetona alcançou-se a mais elevada eficiência de extração para a maioria dos 17 HPAs do solo em relação a todos os solventes testados.

Na avaliação do branco, ou seja, uma análise completa utilizandose apenas o solo calcinado como matriz na extração, não foi identificado nenhum interferente do solvente ou do solo que poderiam coeluir com os compostos de interesse.

A Tabela 4 mostra a concentração dos 17 HPAs nas amostras de solo aplicando-se o método proposto. A determinação dos HPAs nas amostras de solo contendo 50\% de matéria orgânica serviu para avaliar a biodegradação destes compostos durante um processo de vermicompostagem.

Estas concentrações determinadas fazem parte de um projeto de biorremediação de solos contaminados por HPAs, em que o principal objetivo é verificar o comportamento de degradação destes compostos ao longo de um processo de vermicompostagem, utilizando a minhoca Eisenia foetida, vulgarmente conhecida como minhoca vermelha da Califórnia ou minhoca de esterco. Durante o processo adicionouse matéria orgânica ao solo numa proporção de 25, 50, 60 e 75\%, utilizando-se como fonte de matéria orgânica o esterco bovino. Neste trabalho apresentam-se os resultados utilizando-se 50\% de esterco (matéria orgânica), como forma de exemplo.

\section{CONCLUSÕES}

Os 17 HPAs podem ser bem separados e determinados por CLAE com detector UV-Vis e fluorescência usando uma coluna C-18 e misturas acetonitrilina-água como fase móvel. O tempo da corrida cromatográfica é de $20 \mathrm{~min}$. A extração por ultrassom foi escolhida em 


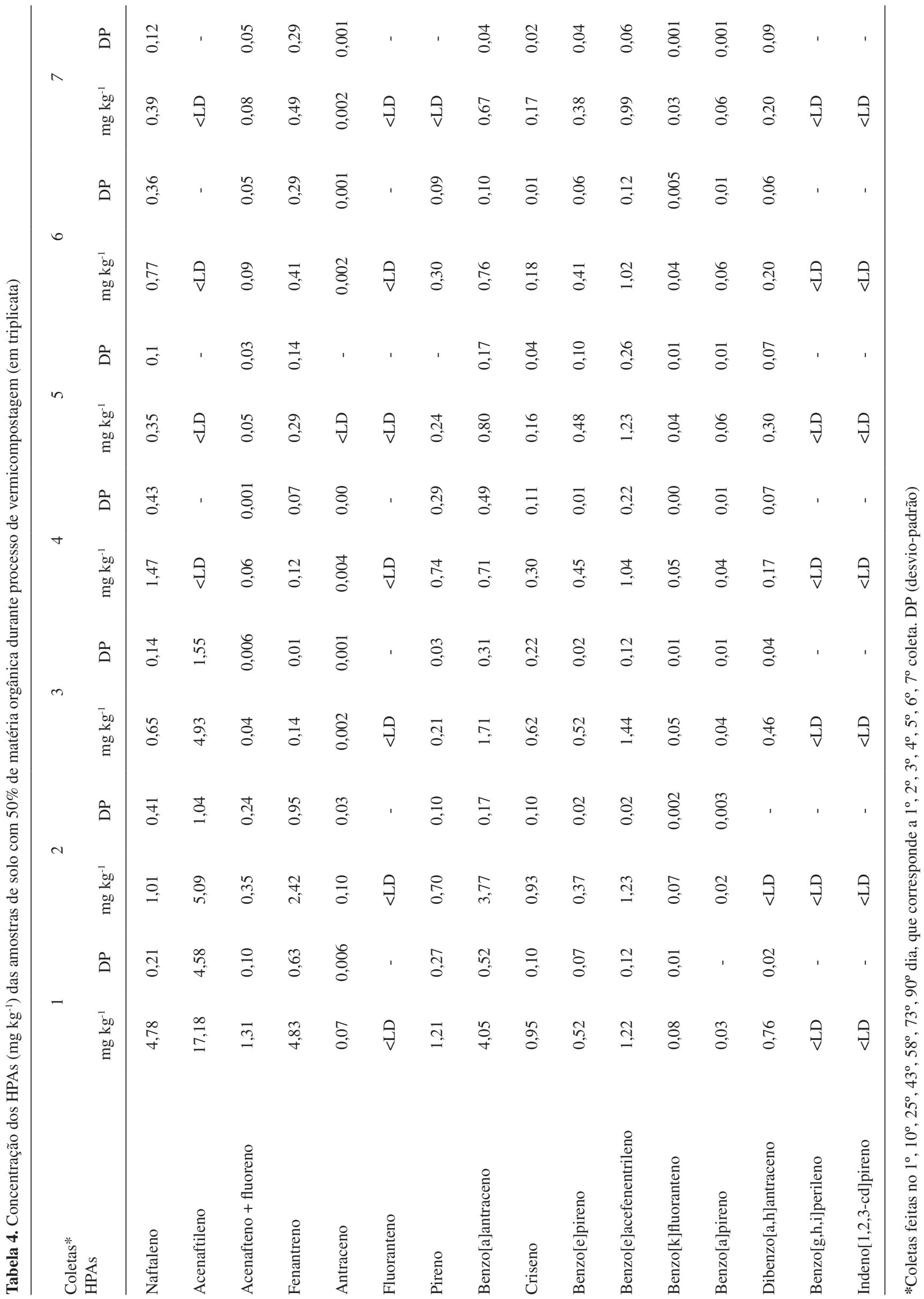


relação à extração por Soxhlet, porque apresentou alta eficiência de extração, baixo custo operacional e fácil operação, além de minimizar o uso de solvente e não levar a perdas por evaporação. A acetona foi o melhor solvente entre os três avaliados para a extração dos 17 HPAs em solo, seguida do metanol e da acetonitrila.

Finalmente, pode ser mencionado que a metodologia proposta pode ser aplicada para o controle dos 17 HPAs em amostras de solo com características próximas às do solo estudado.

\section{REFERENCIAS}

1. Pereira Netto, A. D.; Moreira, J. C.; Dias, A. E. X. O.; Arbilla G.; Ferreira, L. F. V.; Oliveira, A. S.; Barek, J.; Quim. Nova 2000, 23, 765.

2. Gorshkov, G.; Marinaite, I. I.; Baram, G. I.; Sokov, I. A.; J. Anal. Chem. 2003, 58, 768 .

3. Verweij, F.; Booij, K.; Satumalay, K.; van der Molen, N.; van der Oost, R.; Chemosphere 2004, 54, 1675.

4. Codina, G.; Vaquero, M. T.; Comellas, L.; Broto-Puig, F.; J. Chromatogr., A 1994, 673, 21.

5. Berset, J. D.; Ejem, M.; Holzer R.; Lischer P.; Anal. Chim. Acta 1999, 383, 263.

6. Baird, C.; Química Ambiental, 2a ed., Bookman: Porto Alegre, 2002.

7. Tomaniová, M.; Hajslová, J.; PavelkaJr., J.; Kocourek, V.; Holadová K.; Klímova, I.; J. Chromatogr., A 1998, 827, 21.

8. Koh, C.-H.; Khim, J. S.; Kannan, K.; Villeneuve, D. L.; Senthilkumar, K.; Giesy, J. P.; Environ. Pollut. 2004, 132, 489.

9. Rhodes, S.; Farwell, A.; Hewitt, L. M.; MacKinnon, M.; Dixon, D. G.; Ecotoxicol. Environ.Saf. 2005, 60, 247.

10. Quantin, C.; Joner, E. J.; Portal, J. M.; Berthelin, J.; Environ. Pollut. $\mathbf{2 0 0 5}, 134,315$.
11. Utsumi, A.; Nakashima, A.; Ando, K.; Kizu, R.; Hayakama K.; Anal. Sci. 1998, 14, 845.

12. Miège, C.; Dugay, J.; Hennion, M. -C.; J. Chromatogr., A 1998, 823, 219.

13. Obuchi, A.; Aoyama, H.; Ohi, A.; Ohuchi, H.; J. Chromatogr. 1984, 312, 241.

14. Anyakora, C.; Ogbeche, A.; Palmer, P.; Coker, H.; J. Chromatogr., A 2005, 1073, 323.

15. Moret, S.; Conte, L. S.; J. Chromatogr., A 2000, 882, 245.

16. Fedotov, P. S.; Bauer, C.; Popp, P.; Wennrich, R.; J. Chromatogr., A 2004, 1023, 305 .

17. Hawthorne, S. B.; Grabanski, C. B.; Martin, E.; Miller, D. J.; J. Chromatogr., A 2000, 892, 421.

18. Song, Y. F.; Jing, X.; Fleischmann, S.; Wilke, B.-M.; Chemosphere 2002 , 48, 993.

19. Saim, N. ; Dean, J. R.; Abdullah, Md. P.; Zakaria, Z.; J. Chromatogr., A 1997, 791, 361 .

20. Reimer, G.; Suarez, A.; J. Chromatogr., A 1995, 699, 253.

21. Fernéndez-Pérez. V.; Luque de Castro, M. D.; J. Chromatogr., A 2000, 902, 357.

22. Banjoo, D. R.; Nelson, P. K.; J. Chromatogr., A 2005, 1066, 9.

23. Sun, F.; Littlejohn, D.; Gibson, M. D.; Anal. Chim. Acta 1998, 364, 1.

24. Long, L. G.; Winwfordder, J. D.; Anal. Chem. 1983, 55, 712.

25. http://www.epa.gov/sw.846/pdfs/8310.pdf, acessada em Setembro 2009.

26. http://www.varianinc.com/media/sci/apps/lc07.pdf, acessada em Setembro 2009

27. Andrade, S. J.; Dissertação de Mestrado, Universidade Estadual Paulista Júlio de Mesquita Filho, Brasil, 2000. 